Open Access

\title{
Chemical and Immunochemical Characterization of Polysaccharides of Sasa Veitchii Leaves
}

\author{
Masamichi Tsuboi ${ }^{\mathrm{a}, \mathrm{b}}$, Kazuo Takeshita ${ }^{\mathrm{a}}$, Masato Kanamori ${ }^{\mathrm{a}}$, Kazuyuki Umemura $^{\mathrm{c}}$, \\ Kazutoshi Ogawa, ${ }^{\mathrm{c}}$ Natsuko Akachi ${ }^{\mathrm{a}}$, Mia Yoshida ${ }^{\mathrm{a}, \mathrm{d}}$, Naohito Ohno*,d
}

\author{
${ }^{a}$ Hoshi Pharmaceutical Co., Ltd., 7-22-17 Nishigotanda, Shinagawa-ku, Tokyo, 141-0031, Japan; ${ }^{b}$ University of Tokyo; \\ ${ }^{c}$ Department of Environmental Science, College of Science and Engineering, Iwaki Meisei University, 5-5-1 Chuodai- \\ Iino, Iwaki 970-8551, Japan; ${ }^{2}$ Laboratory for Immunopharmacology of Microbial Products, School of Pharmacy, Tokyo \\ University of Pharmacy and Life Sciences, 1432-1 Horinouchi, Hachioji, Tokyo 192-0392, Japan
}

\begin{abstract}
A hot water extract of Sasa veitchii is a health-promoting food in general use. To analyze the structure and function of the polysaccharide fraction of the extract, a macromolecular fraction was obtained by dialysis (HMF) and two polysaccharide fractions (NPS and APS) were separated by DEAE-Sephadex chromatography. HMF strongly reacted with human sera and immunoglobulin preparations. All of the IgM, IgG, and IgA classes of antibodies were reacted with HMF. Comparing the reactivity of NPS and APS, NPS showed significantly stronger reactivity to the sera. From physicochemical analysis, their molecular weights are 20,000 and 8,000, respectively. Sugar analyses of the acid hydrolysates indicated rhamnose, arabinose, xylose, mannose, glucose, and galactose in the molar ratio of $1.0: 2.3: 1.5$ : $3.8: 0.6: 5.3$ for NPS and $1.0: 3.0: 2.6: 0.8: 6.3: 3.0$ for APS, and suggested major differences in the ratio of hexoses. APS also contained $2.6 \%$ galacturonic acid. Methylation analyses suggested that 1 ) both NPS and APS have a highly branched structure, 2) only NPS contains galactofuranose residue at the non-reducing terminal. Partial acid hydrolysis of HMF and subsequent dialysis recovered a high molecular weight fraction, but the resulting product had significantly low immunochemical reactivity. Considering the physicochemical and immunochemical analyses, the major epitope structure of NPS was suggested to be galactofuranose residues. Immunochemical reactivity of the polysaccharide is a key molecular mechanism for the health promoting activity of $S$. veitchii.
\end{abstract}

Keywords: Sasa veitchii, polysaccharide, immunochemical characterization.

\section{INTRODUCTION}

The subject of this article is the chemical as well as immunochemical characterization of a hot water extract of Sasa veitchii (Hoshi's Striped Bamboo Extract, HSBE), which has been used for more than forty years as a healthpromoting food in Japan. It is an industrial product, prepared from bamboo leaves through a unique procedure, in which the foliate substance is halfway decomposed through a controlled treatment with hot water at $103^{\circ} \mathrm{C}$. Some in vivo pharmaceutical studies have shown various HSBE effects. We previously reported its anti-tumor effect in in vivo experiments of a high molecular weight fraction containing polysaccharides of HSBE [1]. The polysaccharide fraction also enhanced the activities of primary host defense against microbial infection [2]. Oral administration of HSBE exhibited anti-ulcer effects [3-5]. Histochemical studies on gastric ulcers suggested that a membrane-stabilizing effect of HSBE might be responsible for the prevention of gastric lesions [4]. We have recently reported that edema caused by inflammatory mediators such as carrageenan, serotonin and

\footnotetext{
*Address correspondence to this auhtor at the Laboratory for Immunopharmacology of Microbial Products, School of Pharmacy, Tokyo University of Pharmacy and Life Sciences, 1432-1 Horinouchi, Hachioji, Tokyo 192-0392, Japan; Tel/Fax: 042-676-5561;

E-mail: ohnonao@ps.toyaku.ac.jp
}

prostaglandin $E_{z}$ was suppressed by the oral administration of HSBE [6]. It was found in those experiments that HSBE reduced inflammatory cytokines such as interleukin- 6 and tumor necrosis factor- $\alpha$. Leaf extracts of genus Sasa spp. have also been reported to show various biological activities, such as antibacterial, antiviral, and antioxidative effects $[7,8]$. It has been shown that lower molecular weight substances such as tricin in genus Sasa extract exhibited anti-human cytomegalovirus activity [9].

Polysaccharides in the extract might have an effect on the host-immune system; however, the molecular entity remains unclear. In the present study, we prepared a high molecular fraction (HMF) by dialysis of HSBE and neutral polysaccharide (NPS) and acidic polysaccharide (APS) fractions by ion-exchange chromatography of HMF. To clarify the structure-activity relationship of NPS and APS, the structures of these polysaccharides were analyzed by chemical methodologies such as determination of sugar constituents by gas chromatography and linkage of sugar residues by methylation analysis. In addition, immunological activities such as reactivity with human sera and gammaglobulin preparation were examined by ELISA, and the antigenic features were analyzed with monoclonal antibodies against plant polysaccharides. On the basis of the evidence obtained here, possible molecular structures have been proposed for NPS and APS. 


\section{MATERIALS AND METHODS}

\section{HSBE}

(Hoshi Kumazasa Bamboo Extract) was supplied by Hoshi Pharmaceutical Co., Ltd. (Tokyo, Japan). HSBE has been used as a herbal medicine derived from the leaves of a particular type of bamboo, Sasa veitchii, in which the foliate substance is halfway decomposed through controlled treatment with hot water at $103^{\circ} \mathrm{C}$. The product is presently sold as a supplement. HSBE is a dark-brown syrup containing $50 \%$ water with a unique flavor and bitter taste, and is weakly acidic (pH4-5).

\section{Enzyme Treatment}

An HMF was prepared by dialysis of HSBE. An aliquot of $\mathrm{HMF}$ solution was treated with ribonuclease at $37^{\circ} \mathrm{C}$ for $48 \mathrm{~h}$, and then protease at $45^{\circ} \mathrm{C}$ for $48 \mathrm{~h}$. After the treatment, the enzyme reaction was terminated by heating. After extensive dialysis, the non-dialyzable fraction was designated as the polysaccharide fraction, PSF (yield, $6.1 \mathrm{~g}$ from $248 \mathrm{~g}$ of HSBE).

\section{Preparation of NPS and APS}

An aliquot of PSF was applied to a DEAE Sephadex A25 column, and eluted with $450 \mathrm{ml}$ of $10 \mathrm{mM}$ phosphate buffer ( $\mathrm{pH}$ 6.0) followed by a $600 \mathrm{ml}$ linear $\mathrm{NaCl}$ gradient $(0$ to $1 \mathrm{M}$ ). The neutral polysaccharide fraction appeared as a single sharp peak in fraction 9, while acidic polysaccharide fractions were eluted as a sharp peak in fraction 93, a smaller peak in 103, and several even smaller peaks. The peak fractions of 9, 93, and 103 appeared in every case irrespective of the growing districts of the bamboo and of their harvesting seasons. We recovered two polysaccharide fractions from the main peak fractions 9 and 93, named NPS and APS, respectively. Yields of NPS and APS were $0.10 \mathrm{~g}$ and $0.076 \mathrm{~g}$ from $0.84 \mathrm{~g}$ PSF. For immunochemical examinations, NPS and APS were prepared directly from HMF.

\section{Preparation of CSBG}

CSBG is a beta-glucan having $\beta-1,3$ and $\beta-1,6$ glucosidic linkages (solubilized candida cell wall beta-glucan). It was prepared according to the method described by Uchiyama and others [10].

\section{Determination of Molecular Weights}

First, 10-30 mg NPS or APS was dissolved in $1 \mathrm{~mL}$ of $0.1 \mathrm{~N} \mathrm{NaOH}$, centrifuged (4000 rpm, $10 \mathrm{~min}$ ) if necessary, and the solution was applied to a Sepharose CL-6B column. The molecular weight was determined by the use of standard dextrans with known molecular weights of 12,000, 50,000 , and 150,000 .

\section{Analysis of Neutral Sugars}

The polysaccharide fraction $(5 \mathrm{mg})$ was hydrolyzed using $2 \mathrm{M}$ trifluoroacetic acid for $4 \mathrm{~h}$ at $100^{\circ} \mathrm{C}$. The hydrolyzate was evaporated to dryness, converted to the corresponding alditol acetate [11], and subsequently analyzed by gas chromatography (GC) using a TC-1701 column from GL Science $\left(60 \mathrm{~m} \times 0.25 \mathrm{~mm}, 220^{\circ} \mathrm{C}\right.$, carrier gas $\left.\mathrm{N}_{2}\right)$.

\section{Analysis for Uronic Acid}

APS (20 mg) was hydrolyzed with $0.5 \mathrm{M}$ sulfuric acid for $14 \mathrm{~h}$ at $100^{\circ} \mathrm{C}$, and the hydrolyzate was neutralized with barium carbonate and filtered. The filtrate was passed through a small Dowex-1 column (acetate form). The acidic sugar eluted from the column with $6 \mathrm{M}$ acetic acid was identified by GC according to the method of Perry and Hulyalkar [12]. The content of uronic acid was measured by the method of Blumenkrants and Asboe-Hansen [13]. GC was carried out on a HITACHI GC-3000 gas chromatograph equipped with a hydrogen flame ionization detector. GCmass spectrometry (MS) analysis was carried out on a Jeol JMS 700 mass spectrometer (EI mode, $70 \mathrm{eV}$ ), using an Aglient Technologies 19091J-413 HP-5 capillary column (30 $\mathrm{m} \times 0.320 \mathrm{~mm}$ ).

\section{Methylation of Polysaccharide Fractions}

NPS was methylated twice by Hakomori's method [14]. APS was reduced twice by sodium borodeuteride with the aid of carbodiimide reagent [15], and the resulting neutral polysaccharide was methylated twice by Hakomori's method [14]. After being hydrolyzed, reduced, and acetylated using the method of Lindberg [11], the partially methylated alditol acetates were analyzed by GC-MS. For GC, a Varian CP-Sil $5 \mathrm{CB}$ column $\left(50 \mathrm{~m} \times 0.25 \mathrm{~mm}, 200^{\circ} \mathrm{C}\right.$, carrier gas $\left.\mathrm{He}\right)$ was used. The molar ratio of partially methylated alditol acetates was calculated from GC peak areas by the method of Sweet et al. [16-18].

\section{ELISA of Anti-NPS and Anti-APS Antibodies}

A 96-well plate (Sumiron MS-8596F; Sumitomo Bakelite Co., Tokyo, Japan) was coated with a antigen candidate $(12.5 \mu \mathrm{g} / \mathrm{mL})$ and the titer was examined with human sera or an immunoglobulin preparation (dilution: $0,10^{4}, 5 \times 10^{3}, 10^{3}$, $5 \times 10^{2}$ ). As the secondary antibody, anti-human $\operatorname{IgG}+\mathrm{M}+\mathrm{A}$, $\mathrm{IgG}$, IgM, or IgA was used. Relative amounts of titers of NPS and APS were determined by the use of CSBG as a standard antigen.

\section{RESULTS}

\section{Immunochemical Characteristics of HMF}

To demonstrate the biological importance of the polysaccharide fraction of Sasa veitchii, the HMF was prepared by dialysis of the hot water extract of $S$. veitchii, HSBE. To test the immunochemical reactivity, an aqueous solution of HMF coated on an ELISA plate was reacted with human sera and detected with antibodies against human IgM, $\mathrm{IgG}$, and IgA. Fungal beta-glucan, CSBG, was used as a reference substance. As shown in Fig. (1), both HMF and CSBG strongly reacted with human sera. Comparing the reactivity between HMF and CSBG, the relative titer to each antibody was different as follows: HMF reacted stronger with the IgM class antibody and CSBG reacted stronger with $\operatorname{IgG}$ and $\operatorname{IgA}$ antibodies. Because of the general understanding that polysaccharides produce the $\operatorname{IgM}$ class antibody easier than other classes, the polysaccharide fraction in HMF might be immunochemically active. In addition, from the viewpoint of class switching of immunoglobulin, regulation of HMF might be different from that of CSBG. 

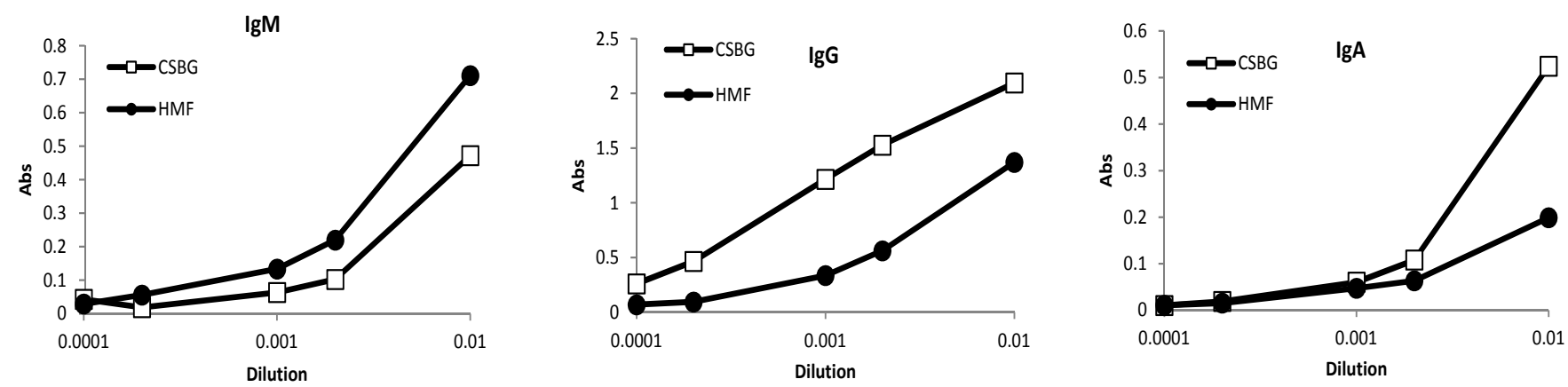

Fig. (1). Reactivity of human sera to HMF. ELISA plate was coated with HMF or CSBG. After blocking non-specific binding sites, diluted sera was added to each well, and then the bound antibody was detected by anti-human $\operatorname{IgM}$, IgG, or IgA.

\section{Preparation of Polysaccharide Fractions}

To prepare the PSF, HMF was treated with enzymes to remove nucleic acids and proteins. Chemical analysis of PSF showed $2.6 \%$ of $\mathrm{N}$ and $5.4 \%$ of ash. To fractionate the PSF, it was applied to a DEAE Sephadex A-25 column equilibrated with $10 \mathrm{mM}$ phosphate buffer. After collecting the pass-through fraction (NPS), the column was eluted with a linear $\mathrm{NaCl}$ gradient ( 0 to $1 \mathrm{M}$ ) in $10 \mathrm{mM}$ phosphate buffer. The elution profile is shown in Fig. (2). It was evident that HMF contained both neutral and acidic polysaccharides. Repeated experiments using various lots of HSBE showed similar elution profiles; therefore, we recovered two polysaccharide fractions from the neutral and acidic fractions and named them NPS and APS, respectively.

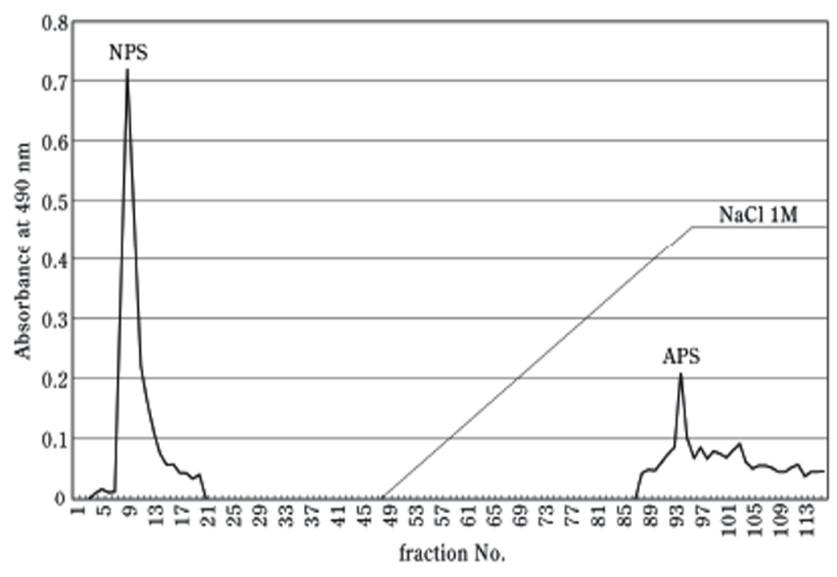

Fig. (2). A representative elution profile of PSF from a DEAESephadex A-25 column equilibrated with $10 \mathrm{mM}$ phosphate buffer. See Materials and methods for experimental details. NPS and APS were prepared from the pass-through and adsorbed fraction, respectively.

Aliquots of NPS or APS were dissolved in $0.1 \mathrm{~N} \mathrm{NaOH}$ and applied to a Sepharose CL-6B column. The molecular weight was assessed using standard dextrans with known molecular weights of 12,000,50,000, and 150,000. A typical elusion pattern is shown in Fig. (3). Both NPS and APS showed symmetrical elution profiles and the average molecular weight of NPS was assessed to be $2.0 \times 10^{4}$ and APS to be $0.8 \times 10^{4}$.

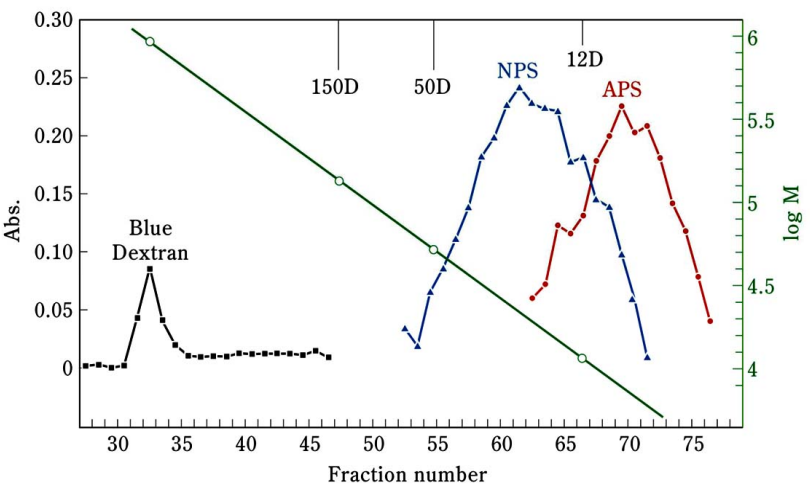

Fig. (3). A representative elution profile of NPS and APS from a Sepharose CL-6B column. See Materials and methods for experimental details. Elution pattern of blue dextran and the regression curve of the estimated molecular weight prepared from the standard dextrans are also shown.

\section{Chemical Characterization of NPS and APS}

Component sugars of NPS and APS were analyzed by alditol acetate derivatives after complete acid hydrolysis and were demonstrated to be rhamnose, arabinose, xylose, mannose, glucose, and galactose in the molar ratio of 1.0 : $2.3: 1.5: 3.8: 0.6: 5.3$ for NPS and $1.0: 3.0: 2.6: 0.8: 6.3$ : 3.0 for APS. APS also contained $2.6 \%$ of uronic acid, which was identified to be galacturonic acid by GC. The results were analyzed from the total carbohydrate content, i.e., the ratio of each component sugar was calculated from the total number as summarized in Fig. (4). Comparing NPS and APS, the ratio of pentoses (rha/ara/xyl) was similar, but that of hexoses (man/glc/gal) was significantly different, i.e., major hexoses in NPS were mannose and galactose, and those in APS were galactose and glucose, respectively.

Linkage of sugar residues was characterized by methylation analysis (Table 1). For NPS, the rate of nonreducing terminal residues (the sum of trimethyl Ara, Xyl, and Rha, and tetramethyl Glc, Man, and Gal) in Table $\mathbf{1}$ to total residues was $32 \%$, whereas the rate of the branching point was $21 \%$. Since methylation analysis of a polysaccharide should give an equimolecular amount of methylsugars derived from branched and terminal residues, it was concluded that about $10 \%(32 \%-21 \%)$ of the branched residues in NPS escaped methylation analysis. For APS, about $30 \%$ of branched residues seemed to resist 
Table 1. Methylation analysis of NPS and APS

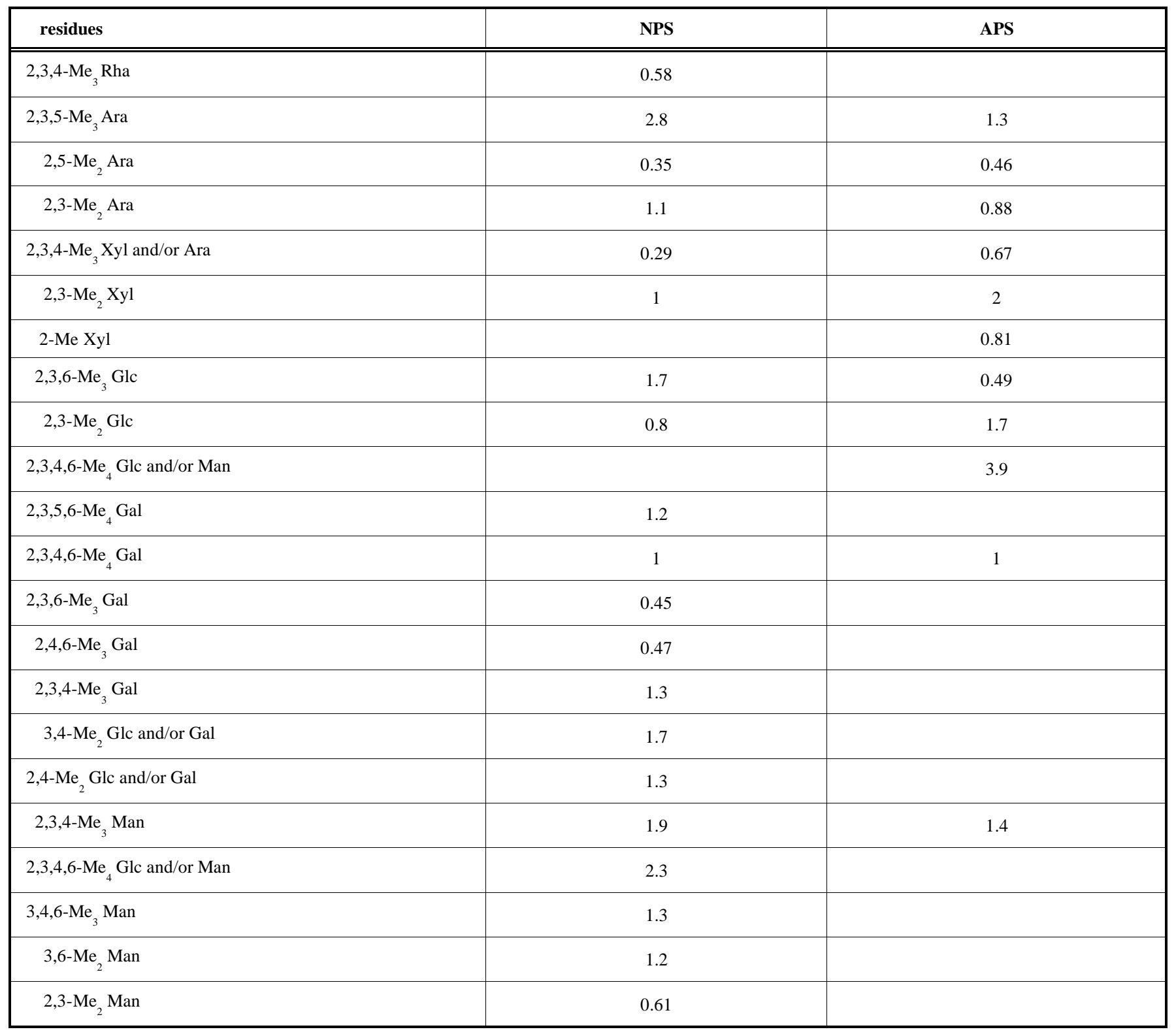

methylation,. The results of methylation analyses showed that both NPS and APS might be highly branched (Table 2). From these findings, the tentative structures of NPS and APS are proposed in Fig. (5).

Table 2. Comparison of architecture of NPS and APS as revealed from methylation analyses

\begin{tabular}{|l|c|c|}
\hline & \multicolumn{2}{|c|}{$\begin{array}{c}\text { Molar ratio calculated from the data in } \\
\text { Table 1 (\%) }\end{array}$} \\
\hline \hline Residues of & NPS & APS \\
\hline Non-reducing terminal & $8.2(32)$ & $6.9(33)$ \\
\hline Linear & $9.6(37)$ & $6.9(33)$ \\
\hline Branching point & $5.6(21)$ & $0.8(4)$ \\
\hline In Core (not detected) & $2.6(11)$ & $6.1(29)$ \\
\hline
\end{tabular}

\section{Immunochemical Characterization of NPS and APS}

As shown in Fig. (1), HMF reacted with antibodies in human sera, and all the classes of $\operatorname{IgM}, \operatorname{IgG}$, and $\operatorname{IgA}$ were included. The high titer of IgM suggested that the polysaccharide component is at least in part the major antigen. To confirm the contribution of the polysaccharide part as an antigen, the reactivity of HMF, NPS, and APS to human sera was compared. All these antigens were coated on ELISA plates and the reactivity was compared by adding serially diluted sera and was detected by anti-human IgM, anti-human IgG, and anti-human IgA. As shown in Fig. (6a, $\mathbf{6 b}$, and $\mathbf{6 c}$ ), human sera reacted with both NPS and APS, but the reactivity was significantly high in NPS detected with all three classes of $\operatorname{IgM}, \operatorname{IgG}$, and $\mathrm{IgA}$.

A similar experiment was performed using a gammaglobulin preparation for clinical use (IVIg) and human sera from 20 volunteers. The results of IVIg were quite similar 
Fig. (6d). Fig. (7) shows the reactivity of sera from individuals, and it was found that all the sera reacted with HMF, NPS, and APS, but the titer was significantly different.

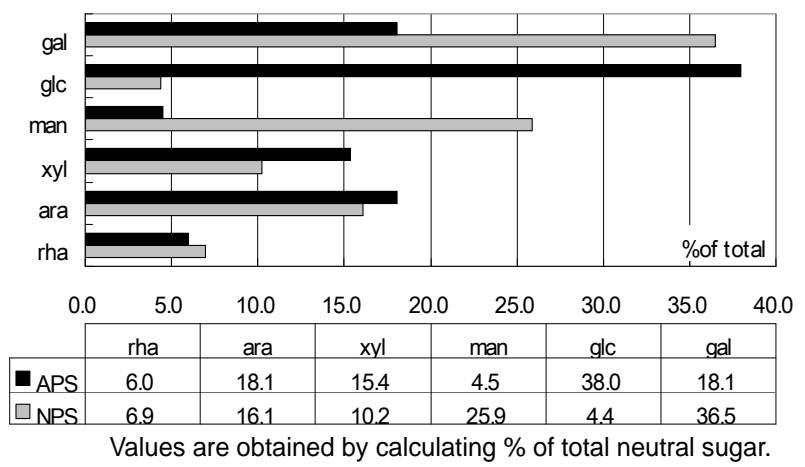

Fig. (4). Rate of component sugars in NPS and APS, calculated from GC analysis of alditol acetate derivatives.

To characterize the immunochemical properties more precisely, the reactivity was demonstrated by a competitive ELISA assay using HMF, NPS, and APS as soluble antigens (Fig. 8).

Using an HMF-coated plate with anti-IgG antibody, HMF and NPS inhibited the reaction almost equally, and
APS showed only slight inhibition. Similar results were obtained using an NPS-coated plate. With an APS-coated plate, 1) reactivity to the sera was low, as assessed by the maximum absorbance, 2) the inhibitory activity of NPS was also high, whereas 3 ) the activity of APS was low. From these findings, APS might have weak antigenicity. In addition, the inhibition rate of APS, detected by anti-human IgG was significantly low compared with that by anti-human IgM. Overall, NPS is suggested to be the major antigenic substance in the extract. In addition, the antigenic substance of APS reacted only with the IgM class antibody, suggesting that the epitope of NPS and APS would be partly different.

In the previous section, it was evident that the major difference in the structure of NPS and APS is the component of hexoses, i.e., mannose is dominant in NPS and glucose in APS. Galactose is also rich in NPS. Configuration of these residues was not determined in this study; however, in the preliminary study, reactivity of HMF was tested against various plant lectins, i.e., HMF bound to PSA (from Pisum sativum), LCA (from Lens culinaris), Calsepa (from Calystegia sepium), and GSL-I-B4 (from Griffonia simplicifolia). PSA, LCA, and Calsepa, all recognized alphalinked mannose, and GSL-I-B4 recognized alpha-linked galactose, suggesting that Man and Gal residues in $\mathrm{HMF}$ have an alpha configuration.
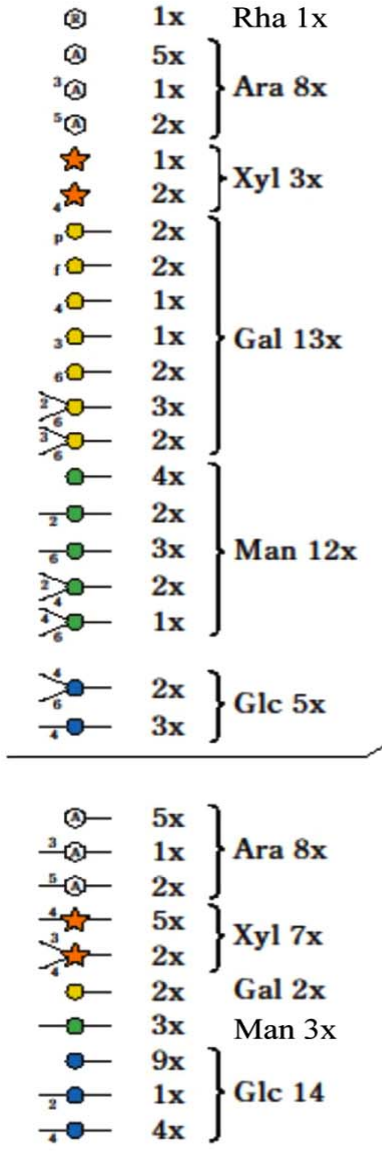
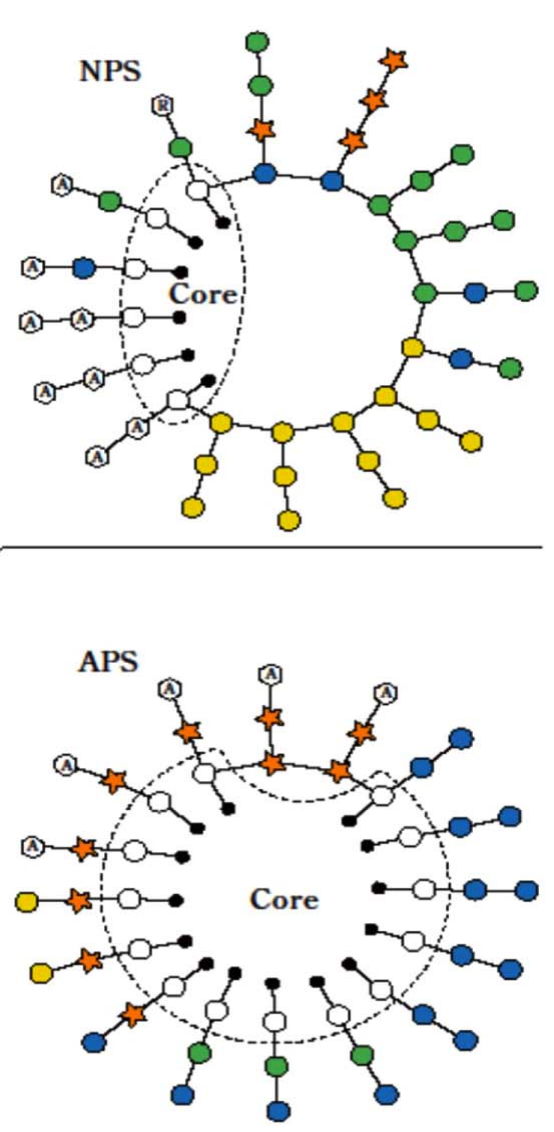

Fig. (5). Proposed architecture of NPS and APS. Each symbol represents a sugar residue, and the name of the sugar is given on the left side. The "core" is the portion which could not be methylated by the Hakomori method. 
(a)

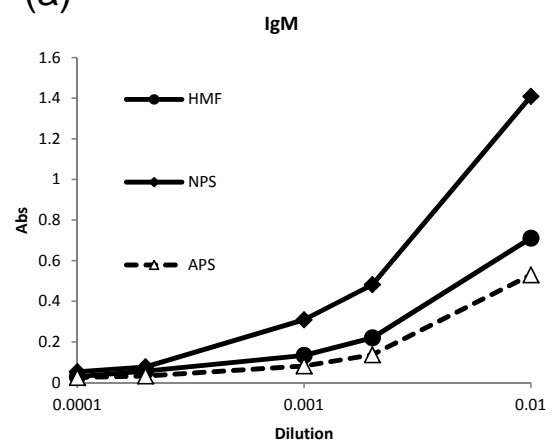

(b)

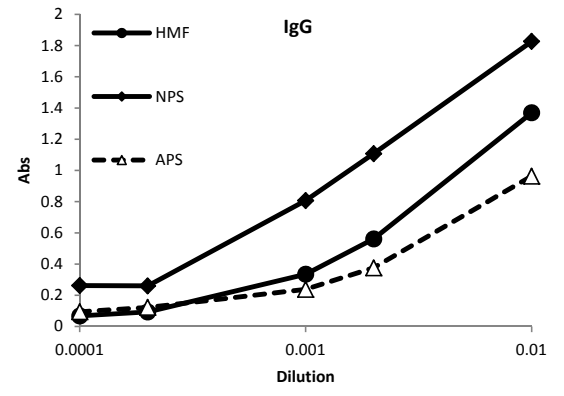

(c)

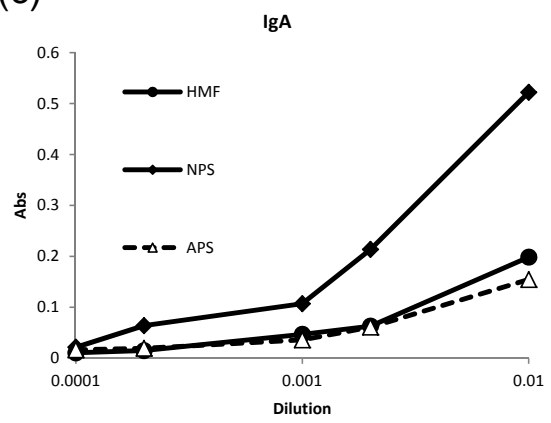

$(d)$

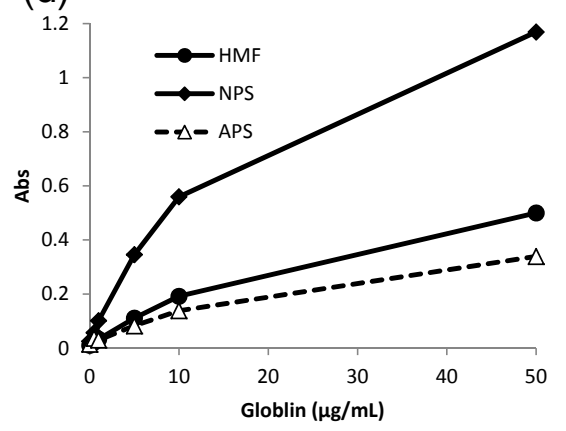

Fig. (6). Reactivity of human sera and immunoglobulin preparation to HMF, NPS, and APS. Reactivity to human sera was detected by antihuman $\operatorname{IgM}(\mathbf{a}), \operatorname{IgG}(\mathbf{b})$, and $\operatorname{IgA}$ (c) antibody. Reactivity to immunoglobulin preparation was detected by anti-human IgG antibody (d).

(a)

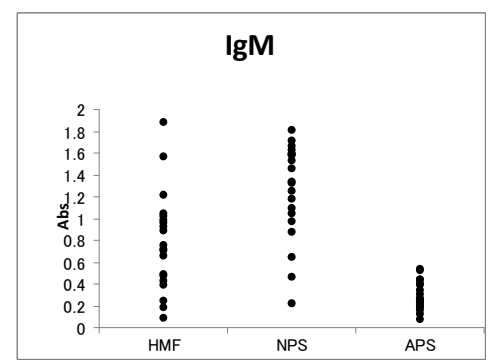

(b)

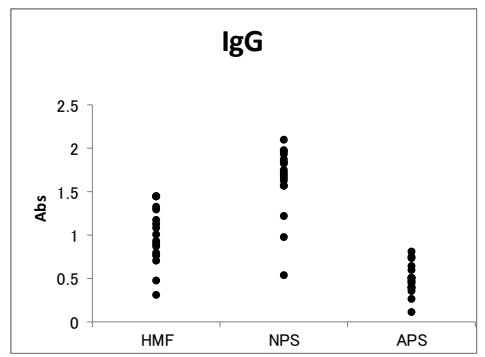

(c)

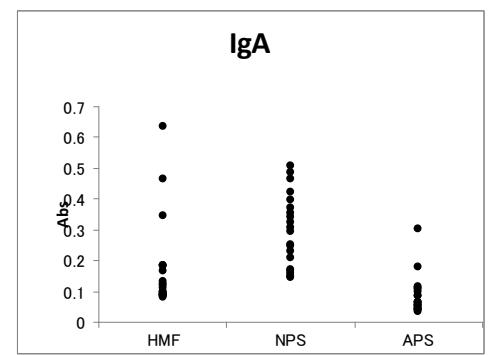

Fig. (7). Anti-HMF, NPS, and APS reactivity of sera from volunteers. Reactivity to human sera was detected by anti-human IgM (a), IgG (b), and $\operatorname{IgA}(\mathbf{c})$ antibodies.

\section{Further Immunological Characterization of NPS and APS}

To demonstrate the antigen structure, several standard polysaccharide antigens and commercially available monoclonal antibodies against plant polysaccharides were analyzed. Figs. (9a and 9b) show the inhibition of antibody binding to HMF, NPS, and APS by mannan (CAWS) and xylan, respectively. It was clearly demonstrated that commercially available xylan competed with HMF and NPS. To further analyze the epitope structure, monoclonal antibodies, CCRC-M133 and CCRC-M138, were applied. Epitopes of M133 and M138 were arabinogalactan and xylan, respectively. In the preliminary investigation, M133 did not react with HMF at all (data not shown). Fig. (9c) shows the competition of M138 to HMF with several fractions. Reactivity of M138 most strongly competed with commercial xylan. In addition, all the fractions of HMF, NPS, and APS competed with the antibody binding. These findings strongly suggested that xylan moiety, at least in part, showed antigenicity.
In the results of methylation analysis, it was evident that a large proportion of arabinofuranose residue, 2,3,5- $\mathrm{Me}_{3}$ Ara and 2,5- $-\mathrm{Me}_{2}$ Ara, and galactofuranose residue, 2,3,5,6- $\mathrm{Me}_{4}$ Gal, was present in NPS, especially on the non-reducing terminus (Table 1). To demonstrate the contribution of furanose residues on antigenicity, mild acid hydrolysis was performed on HMF and the immunoreactivity was compared. As shown in Fig. (10), the resulting substance lost significant antigenicity both to HMF and NPS. Comparing the composition of furanose residues in NPS and APS, galactose residue is present only in NPS. From these findings, galactofuranose might be the major epitope of NPS.

\section{DISCUSSION}

Illness types change due to the development of society, i.e., increased risk of new infectious diseases due to people traveling all over the world, changing eating habits from traditional Japanese to western style increases the risk for metabolic syndrome, and the development of new medicines for various diseases increases the mean lifespan, resulting in 
an older society. Herbal medicine has long been clinically applied throughout the world for the treatment of various illnesses. In addition, combination use of western modern medical treatment and traditional herbal medicine has recently been emphasized for the treatment of chronic intractable diseases.

The function of polysaccharide components in medicinal plants has been extensively investigated all over the world and evidence has suggested their pharmacological importance. For example, anti-ulcer polysaccharides have been characterized from various plants, such as Panax ginseng, Bupleurum falcatum, Maytenus ilicifolia, Lycium barbarum, and Decalepis hamiltonii. Sakurai et al. precisely characterized the polysaccharides from B. falcatum and suggested the distribution of these polysaccharides in the
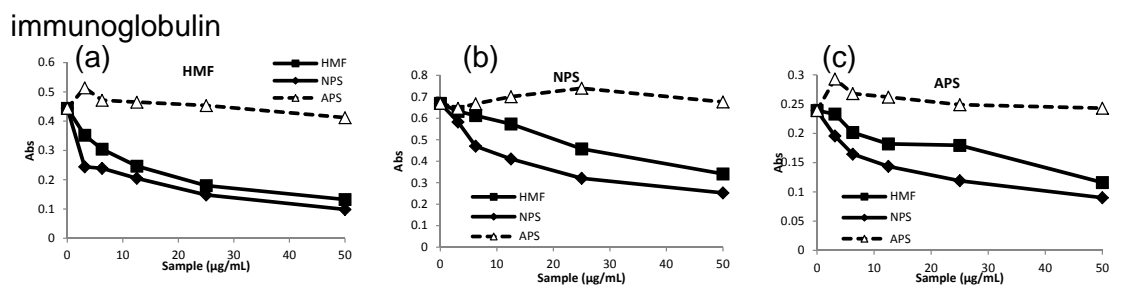

\section{Serum-lgG}

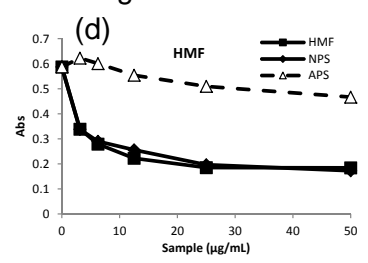

1.4. (e)
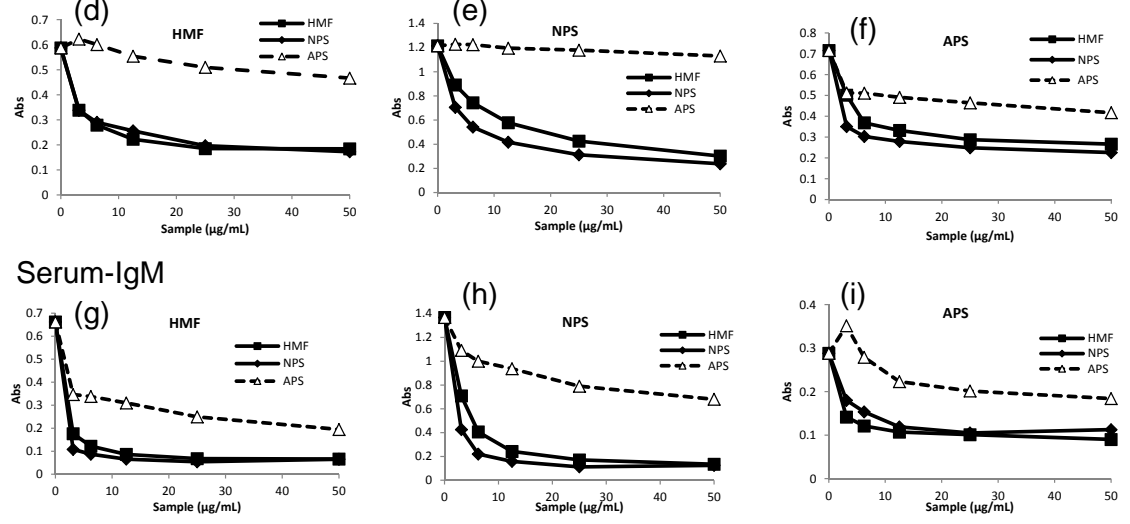

Fig. (8). Assessment of the specificity of immunoreactivity by adding soluble antigen. Each ELISA plate was coated with HMF (a, d, g), NPS $(\mathbf{b}, \mathbf{e}, \mathbf{h})$, and APS $(\mathbf{c}, \mathbf{f}, \mathbf{i})$, respectively. Immunoglobulin $(\mathbf{a}, \mathbf{b}, \mathbf{c})$ or human sera $(\mathbf{d}, \mathbf{e}, \mathbf{f}, \mathbf{g}, \mathbf{h}, \mathbf{i})$ was added to each well in the presence or absence of various concentrations of soluble HMF, NPS, or APS, and detected with anti-human $\operatorname{IgG}(\mathbf{a}, \mathbf{b}, \mathbf{c}, \mathbf{d}, \mathbf{e}, \mathbf{f})$ or $\operatorname{IgM}(\mathbf{g}, \mathbf{h}, \mathbf{i})$.
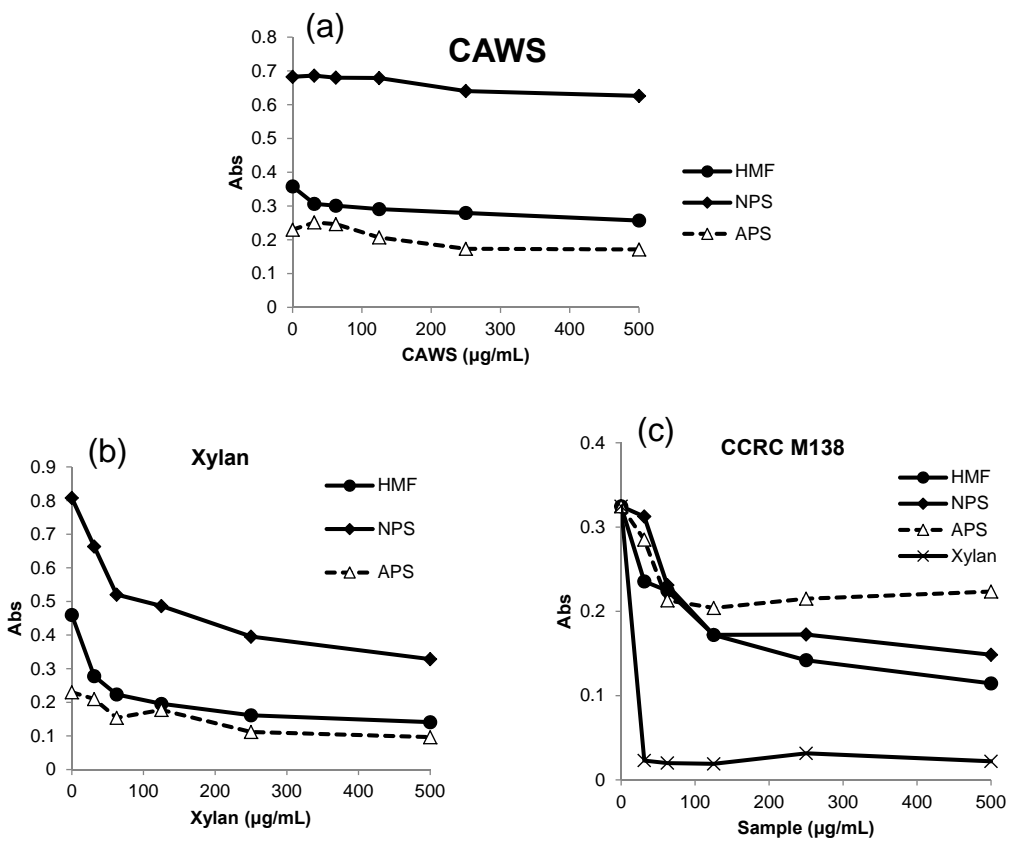

Fig. (9). Examination of antigenic specificity of HMF, NPS, and APS assessed by mannan (a) or xylan (b, c). ELISA plate was coated with HMF, NPS, or APS, and human sera added in the presence or absence of mannan (a, CAWS) or xylan (b), and binding of human sera to the ELISA plate was detected by human anti-IgG. ELISA plate was coated with xylan, and monoclonal anti-xylan antibody and CCRC M138 were added in the presence or absence of HMF, NPS, APS, or xylan, and detected with anti-mouse IgG. 

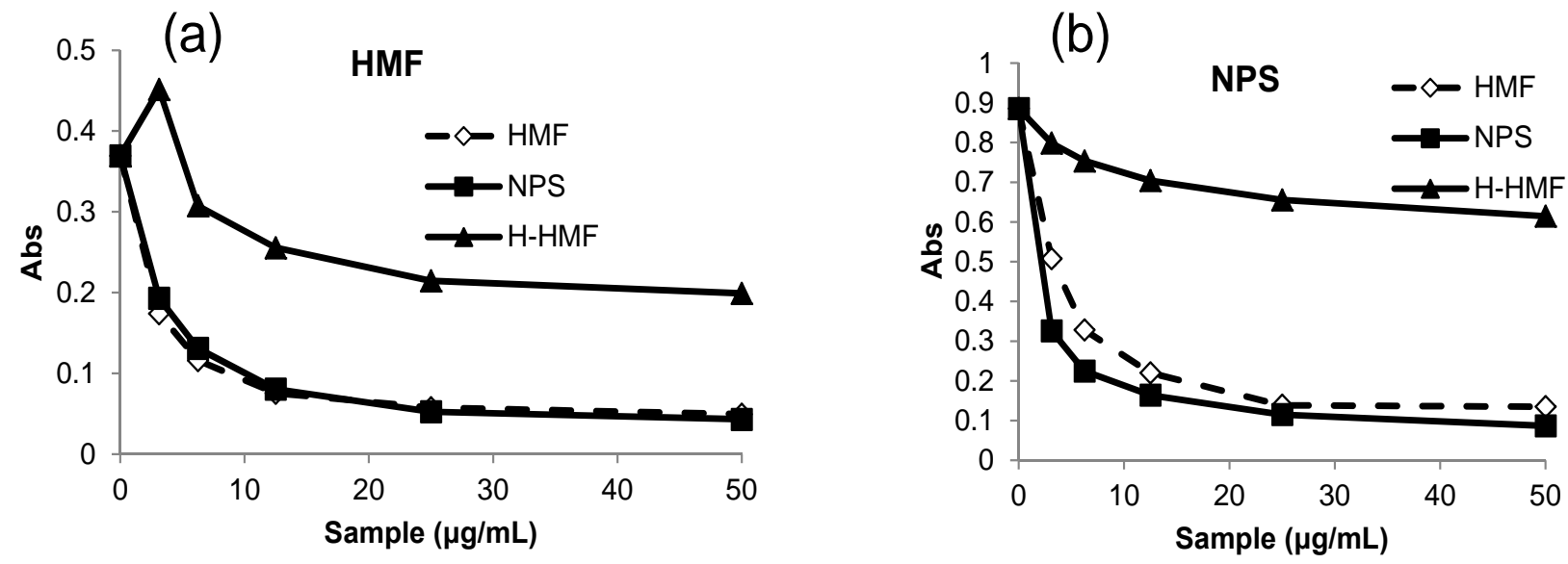

Fig. (10). Characterization of the major epitope of HMF by mild acid hydrolysis. ELISA plate was coated with HMF(a) or NPS(b) and human sera added in the presence or absence of HMF, NPS, or partially acid hydrolyzed HMF (H-HMF), and detected by anti-human IgG.

liver and Peyer's patch by oral administration [19]. Srikanta characterized the molecular mechanisms of $D$. hamiltonii due to the combination of free radical scavenging, antioxidants, anti-H pylori, inhibition of $\mathrm{H}+$ and $\mathrm{K}+$-ATPase and gastric mucosal protective activities [20]. Currier et al. reported that a neutral arabinogalactan from larch enhanced the activity of immune cells, natural killer cells, as well as hemopoietic cells in the bone marrow and spleen [21].

We have previously demonstrated that HSBE shows various pharmacological activities, such as antitumor activity, antimicrobial activity, antiulcer activity, and antiinflammatory activity. HSBE is the hot water extract of Sasa and contains several chemical constituents having various chemical structures. HSBE is now known to have two polysaccharide fractions, NPS and APS, each of which has a very complex chemical structure, and no unequivocal chemical formula has been assigned. It has been established, however, that they each have a very complicated hard core, with a high number, and a great variety, of oligosaccharide side chains (Fig. 5). Because of the variety of such sidechains, HSBE can bind to many antibodies, lectins, and/or antibodies to initiate many different immune reactions. This reactivity may differ according to the individuality of the patient, as may be suggested from our experimental results, shown in Fig. (7) above. It may be suggested further that such a core with many oligosaccharide side-chains might be a protocol for herbal medicine that can initiate many immunological reactions.

\section{CONFLICT OF INTEREST}

The authors confirm that this article content has no conflicts of interest.

\section{ACKNOWLEDGEMENT}

Declared none.

\section{REFERENCES}

[1] Kanamori M, Sakamoto M, Ogata S, Yamamoto A. Studies on antitumor effects of extracts of bamboo grass. J Kyorin Med Soc 1973; 4: 163-7.

[2] Endo N, Onogawa T, Kanamori M. Effects of bamboo extract on the production of factors toward non-specific host defense against microbial infection. J Kyorin Med Soc 1994; 25: 207-13.
[3] Shibata M, Yamatake Y, Sakamoto M, Kanamori M, Takagi K, Okabe S. Pharmacological studies on bamboo grass. Folia Pharmacol Japon 1975; 71, 481-90.

[4] Otani K, Yanaura S, Yuda Y, et al. Histo-chemical studies on the anti-ulcer effect of bamboo grass in rats. Int J Tissue React 1990; 12: 319-32.

[5] Hirose K, Onishi H, Sasatsu M, et al. In vivo evaluation of kumazasa extract and chitosan films containing the extract against deep skin model in rats. Biol Pharm Bull 2007; 30: 2406-11.

[6] Akasaki N, Sasaki Y, Takeda H, et al. Anti-inflammatory effects of kumazasa water extract. Pharmacometrics 2011; 80: 35-42.

[7] Sakagami H, Amano S, Kikuchi H, et al. Antiviral, anitibacterial and vitamin-synergized radical-scavenging activities of Sasasenanensis rehder extract. In Vivo 2008; 22: 471-6.

[8] Hasegawa T, Tanaka A, Hosoda A, Tkano F, Ohta T. Antioxidant C-glycosyl flavones from the leaves of Sasakurilensis var. gigantean. Phytochemistry 2008; 69: 1419-24.

[9] Sakai A, Watanabe K, Koketsu M, et al. Anti-human cytomegalovirus activity of constituents from Sasaalbomarginata(Kumazasa in Japan). Antiviral Chem Chemother 2008; 19: $125-32$.

[10] Uchiyama M, Ohno N, Miura NN, et al. Solubilized cell wall $\beta$ Glucan, CSBG, Is an epitope of candida immune mice. Biol Pharm Bull 2000; 23: 672-6.

[11] Lindberg B. Methods in enzymology Vol.28. In: Ginsburg V. Ed. Newyork: Academic Press 1972; pp.178-19

[12] Perry MB, Hulyalkar RK. The analysis of hexuronic acids in biological materials by gas-liquid partition chromatography. Can $\mathrm{J}$ Biochem 1965; 43: 573-84

[13] Blumenkrants N, Asboe-Hansen G. New method for quantitative determination of uronic acids. Anal Biochem 1973; 54: 484-9

[14] Hakomori S. A rapid permethylation of glycolipid and polysacchride catalized by methylsulforide carbanion in dimethyl sulforide. J Biochem 1964; 55: 205-8.

[15] Taylor RL, Conrad HE. Stoichiometric depolymerization of polyuronides and glycosaminoglycuronans to monosaccharides following reduction of their carbodiimide-activated carboxyl groups. Biochemistry 1972; 11: 1383-8.

[16] Sweet DP, Shapiro RH, Albersheim P. Quantitive analysis by various G.L.C, response factor theories for partially methylated and partially ethylatrd alditol acetate. carbohyr Res 1975; 40: 217-25.

[17] Carpita NC, Shea EM. Analysis of Carbohydrates by GLC and MS. In: Biermann CJ, McGinis GD. Eds. Boca Raton: CRC Press 1989; pp.157-216.

[18] Ogawa K, Yamaura M, Maruyama I. Isolation and Identification of 3-O-Methyl-D-galactose as a constituent of nutral polysaccharide of Chlorela vulgaris. Biosci Biotechnol Biochem 1994; 58: 942944.

[19] Sakurai M H, Matsumoto T, Kiyohara H, Yamada H. Detection and tissue distribution of anti-ulcer pectic polysaccharides from Bupleurum falcatum by polyclonal antibody. Planta Med 1996; 62(4): 341-6.

[20] Srikanta BM, Siddaraju MN, Dharmesh SM. A novel phenol-bound pectic polysaccharide from Decalepis hamiltonii with multi-step 
ulcer preventive activity, World J Gastroenterol 2007; 13(39): 5196-207

[21] Currier NL, Lejtenyi D, Miller SC. Effect over time of in-vivo administration of the polysaccharide arabinogalactan on immune and hemopoietic cell lineages in murine spleen and bone marrow. Phytomedicine 2003; 10(2-3): 145-53

(C) Tsuboi et al.; Licensee Bentham Open.

This is an open access article licensed under the terms of the Creative Commons Attribution Non-Commercial License (http://creativecommons.org/licenses/by-nc/3.0/) which permits unrestricted, non-commercial use, distribution and reproduction in any medium, provided the work is properly cited. 